\title{
On spectral asymptotics and bifurcation for Carrier equations with odd superlinear term
}

\section{Yu Zhang ${ }^{1} \cdot$ Baoqiang Yan ${ }^{1}$}

Received: 29 February 2020 / Accepted: 30 July 2020 / Published online: 1 September 2020

(C) The Author(s) 2020

\section{Abstract}

In this paper, we consider the existence of eigenvalues and relative eigenfunctions for Carrier equations and present spectral asymptotics and bifurcation concerning the eigenvalues of some related elliptic linear problem.

Keywords Carrier equations · Liusternik-Schnirelmann (LS)theory · Eigenvalue · Eigenfunction

Mathematics Subject Classification 34B10 $\cdot 34$ B16

\section{Introduction}

In this paper, we consider the following nonlocal elliptic problem

$$
\begin{array}{r}
-\left(a+b \int_{\Omega}|u(x)|^{2} d x\right) \Delta u+f(x, u)=\lambda u, \quad x \text { in } \Omega, \\
u(x)=0, \quad x \text { on } \partial \Omega,
\end{array}
$$

where $\Omega \subseteq \mathbb{R}^{N}(N \geq 1)$ is a smooth and bounded domain, and $a>0, b>0$.

Problem (1.1) is related to the stationary analogue of the equation

$$
u_{t t}-\left(a+b \int_{0}^{\pi}|u|^{2} d x\right) u_{x x}=0
$$

proposed by Carrier [6] which describes the vibration of the elastic string when the change of the tension is not very little.

This article is part of the topical collectiondedicated to Prof. Dajun Guo for his 85th birthday, edited by Yihong Du, Zhaoli Liu, Xingbin Pan, and Zhitao Zhang.

This work is supported by the National Natural Science Foundation of China(61603226) and the Fund of Natural Science of Shandong Province (ZR2018MA022).

Baoqiang Yan

yanbqcn@aliyun.com

1 School of Mathematics and Statistics, Shandong Normal University, Jinan 250014, China 
For the case $b=0$, problem (1.1) is changed as

$$
\begin{array}{r}
-a \Delta u+f(x, u)=\lambda u, \quad x \text { in } \Omega, \\
u(x)=0, \quad x \text { on } \partial \Omega .
\end{array}
$$

and some authors considered the spectral asymptotics, bifurcation and the normalized solutions for problem (1.2) via variational method, see [5, 7, 8, 18-21, 23-25].

Since $-\left(a+b \int_{\Omega}|u(x)|^{2} d x\right) \Delta u$ is lack of variational structure, it is difficult to study problem (1.1) via variational method. Some authors focus on the existence of positive solutions for problem (1.1) or some generalized cases only via the theory of topological theory, the method of lower and upper solutions and pseudomontone operators theory when $\lambda$ is fixed, see [1-3, 9-13, 26-28]. For examples in [26] and [27], authors considered the following problem

$$
\begin{array}{r}
-a\left(\int_{\Omega}|u(x)|^{\gamma} p d x\right) \Delta u=\lambda u^{q}+u^{p}, \quad x \text { in } \Omega, \\
u(x)=0, \quad x \text { on } \partial \Omega,
\end{array}
$$

where $\gamma \geq 1, \quad 0<q \leq 1, \quad p>1, \quad a: \mathbb{R} \rightarrow(0,+\infty)$ is a continuous function with $\inf _{t \in \mathbb{R}} a(t)=a(0)>0$; using the theory of fixed point index on cone, the authors proved that there exist $0<\lambda_{1} \leq \lambda_{2}$ such that (1.3) has no positive solutions for $\lambda>\lambda_{2}$, at least a positive solution for $\lambda=\lambda_{1}$ and $\lambda_{2}$ and at least two positive solutions for $\lambda \in\left(0, \lambda_{1}\right)$; in [14], combing sub-super and bifurcation methods, the authors showed that there exists a drastic change on the structure of the set of positive solutions when the non-local coefficient grows fast enough to infinity for problem (1.3).

Our aim is to present some results on spectral asymptotics and bifurcation for problem (1.1).

This paper is organized as follows. In Sect. 2, using the Liusternik-Schnirelmann (LS) theory, we obtain, given any $r>0$, the existence of infinitely many eigenvalues $\mu_{n, r}($ $n=1,2, \cdots)$ for problem (1.1) associated with eigenfunctions $u_{n, r}$ satisfying $\int_{\Omega} u_{n, r}^{2}(x) d x=r^{2}$. And then Sect. 3 presents bifurcation and comparison results concerning the eigenvalues of some related linear problems (2.1) $)_{\lambda}$. In Sect. 4, we discuss the asymptotic laws of the eigenvalues $\mu_{n, r}$ of problem (1.1) as $n \rightarrow+\infty$ when $f$ is superlinear at $+\infty$. Our paper was motivated in part by the papers $[7,8,15,16,18,21,22]$.

\section{Existence of the eigenvalues of problem (1.1)}

It is easy to see that problem (1.1) is equivalent to its weak formulation, namely that of finding $u \in W_{0}^{1,2}(\Omega)$ and $\lambda \in R$ such that

$$
\left(a+b \int_{\Omega} u^{2}(x) d x\right) \int_{\Omega} \nabla u \cdot \nabla v d x+\int_{\Omega} f(x, u) v d x=\lambda \int_{\Omega} u v d x
$$

for all $v \in W_{0}^{1,2}(\Omega)$, where $W_{0}^{1,2}(\Omega)$ denote the closure of $C_{0}^{\infty}(\Omega)$ in the Sobolev space $W^{1,2}(\Omega)$ with the scalar product $(u, u)=\int_{\Omega} \nabla u \cdot \nabla u d x$ and the corresponding norm $\|u\|=\left(\int_{\Omega}|\nabla u|^{2} d x\right)^{\frac{1}{2}}$, while $\|u\|_{p}$ denotes the norm of $u \in L^{p}(\Omega)$.

For $r>0$, let 


$$
M_{r}:=\left\{u \in W_{0}^{1,2}(\Omega) \mid \int_{\Omega} u^{2} d x=r^{2}\right\}
$$

and for each $n=1,2, \ldots$, set

$$
K_{n, r}=\left\{K \subseteq M_{r}: K \text { compact, symmetric, } \gamma(K)=n\right\}
$$

where $\gamma(K)$ denotes the genus of $K$. For fixed $r>0$ and for $u \in W_{0}^{1,2}(\Omega)$, define

$$
\Phi(u):=\left(a+b r^{2}\right) \frac{1}{2}\|\nabla u\|_{2}^{2}, \quad \Psi(u):=\int_{\Omega} F(x, u(x)) d x
$$

and

$$
I(u):=\Phi(u)+\Psi(u),
$$

where

$$
F(x, u(x))=\int_{0}^{u(x)} f(x, s) d s .
$$

It is well known that the linear elliptic problem

$$
\begin{array}{r}
-\Delta u=\lambda u, \quad x \text { in } \Omega, \\
u(x)=0, \quad x \text { on } \partial \Omega,
\end{array}
$$

has eigenvalues $\lambda_{1}<\lambda_{2} \leq \cdots \leq \lambda_{n} \leq \cdots$ and the corresponding eigenfunction to $\lambda_{n}$ is $u_{n}$ with $u_{n} \in M_{r}$, see [7]. For each eigenvalue $\lambda_{n}$, multiplying $u_{n}$ and integrating on $\Omega$ for $(2.1)_{\lambda}$, we have

$$
r^{2} \lambda_{n}=\int_{\Omega} u_{n}^{2} d x \lambda_{n}=\int_{\Omega}\left|\nabla u_{n}\right|^{2} d x
$$

Since the set of all eigenfunctions corresponding to $\lambda_{n}$ is a linear space, if we choose $v_{n}$ is a eigenfunction of $\lambda_{n}$ with $\int_{\Omega}\left|v_{n}\right|^{2} d x=1$, then the eigenfunction $u_{n}$ of $\lambda_{n}$ with $u_{n} \in M_{r}$ can be written as $u_{n}=l_{n} v_{n}$. From

$$
r^{2}=\int_{\Omega}\left|u_{n}\right|^{2} d x=\int_{\Omega}\left|l_{n} v_{n}\right|^{2} d x=l_{n}^{2} \int_{\Omega}\left|v_{n}\right|^{2} d x,
$$

we get $l_{n}= \pm r$, i.e.,

$$
u_{n}= \pm r v_{n}, \quad n=1,2, \ldots
$$

which together with (2.2) gives

$$
r^{2} \lambda_{n}=\int_{\Omega}\left|\nabla u_{n}\right|^{2} d x=\int_{\Omega}\left|\nabla\left( \pm r v_{n}\right)\right|^{2} d x=r^{2} \int_{\Omega}\left|\nabla v_{n}\right|^{2} d x
$$

and so

$$
\lambda_{n}=\int_{\Omega}\left|\nabla v_{n}\right|^{2} d x
$$


Now, we introduce (see [4]) the "LS critical levels"

$$
c_{n, r}:=\inf _{K_{n, r}} \sup _{K} 2 I .
$$

The following lemma is needed in our proof.

Lemma 2.1 (See [8]) Let $p: 1 \leq p \leq p_{0}=(N+2) /(N-2)$ (so that $2 \leq p+1 \leq 2^{*}$ ) and let $\beta=\left(N / 2^{*}\right)\left(2^{*}-(p+1)\right)$. Then, for each $\gamma: 0 \leq \gamma \leq \beta$, there exists $c>0$ such that

$$
\|u\|_{p+1}^{p+1} \leq c\|\nabla u\|_{2}^{p+1-\gamma}\|u\|_{2}^{\gamma}
$$

for all $u \in W_{0}^{1,2}(\Omega)$. (Here and henceforth $\|u\|_{p}$ denotes the norm of $u$ in $L^{p}(\Omega)$.)

We will consider the following condition:

$\left(A_{1}\right) f: \Omega \times \mathbb{R} \rightarrow \mathbb{R}$ is continuous, $f(x,-u)=-f(x, u)$ and satisfies

$$
|f(x, u)| \leq c|u|^{p}+d
$$

for some $c, d \geq 0$ and some $0 \leq p<\bar{p}=\min \left\{2^{*}-1,1+4 / N\right\}$.

From the $L S$ theory, we have the following existence result.

Theorem 2.1 Assume $\left(A_{1}\right)$ holds. Then, for given $r>0$, there exists a sequence $\left\{u_{n, r}\right\}$ of (weak) eigenfunctions of (1.1) belonging to $M_{r}$, and such that

$$
2 I\left(u_{n, r}\right)=c_{n, r}
$$

where $c_{n, r}$ is as in (2.4); the eigenvalue $\mu_{n, r}$ corresponding to $u_{n, r}$ satisfies

$$
r^{2} \mu_{n, r}=\left(a+b r^{2}\right)\left\|\nabla u_{n, r}\right\|_{2}^{2}+\int_{\Omega} f\left(x, u_{n, r}\right) u_{n, r} d x .
$$

Proof The proof is divided into three steps.

Step 1. We show that

$$
-\infty<c_{n, r}=\inf _{K_{n, r}} \sup _{K} 2 I<+\infty .
$$

First, $\left(A_{1}\right)$ and Schwarz's inequality imply that

$$
\int_{\Omega}|F(x, u(x))| d x \leq c \int_{\Omega}|u|^{p+1} d x+d\left(\int_{\Omega}|u|^{2} d x\right)^{\frac{1}{2}}
$$

for some new constants $c, d>0$.

Moreover, we use the inequality (2.5) with $\gamma=\beta$ : on setting $2 \alpha=p+1-\beta=(p-1) N / 2,(2.6)$ becomes

$$
\int_{\Omega}|u|^{p+1} d x \leq c^{\prime}\|\nabla u\|_{2}^{2 \alpha}\left(\int_{\Omega} u^{2} d x\right)^{\frac{\beta}{2}} .
$$

Next, from (2.6) and (2.7), for $u \in M_{r}$, we have 


$$
\begin{aligned}
I(u) e & \leq\left(a+b r^{2}\right) \frac{1}{2}\|\nabla u\|_{2}^{2}+\int_{\Omega} F(x, u(x)) d x \\
& \leq\left(a+b r^{2}\right) \frac{1}{2}\|\nabla u\|_{2}^{2}+c \int_{\Omega}|u|^{p+1} d x+d\left(\int_{\Omega}|u|^{2} d x\right)^{\frac{1}{2}} \\
& \leq\left(a+b r^{2}\right) \frac{1}{2}\|\nabla u\|_{2}^{2}+c^{\prime}\|\nabla u\|_{2}^{2 \alpha}\left(r^{\beta}\right)+d r,
\end{aligned}
$$

which together with the compactmess of $K \subset K_{n, r}$ implies that

$$
\sup _{u \in K} 2 I(u)<+\infty .
$$

Finally, from (2.6) and (2.7), for $u \in M_{r}$, we have also

$$
\begin{aligned}
I(u) & \geq\left(a+b r^{2}\right) \frac{1}{2}\|\nabla u\|_{2}^{2}-\int_{\Omega}|F(x, u(x))| d x \\
& \geq\left(a+b r^{2}\right) \frac{1}{2}\|\nabla u\|_{2}^{2}-\left[c c^{\prime} r^{\beta}\|\nabla u\|_{2}^{2 \alpha}+d r\right] .
\end{aligned}
$$

The assumption $p<\min \left\{2^{*}-1,1+4 / N\right\}$ is equivalent to $2 \alpha<2$, which implies that $I$ is bounded below on $M_{r}$ (for each $r$ ).

Consequently,

$$
-\infty<c_{n, r}=\inf _{K_{n, r}} \sup _{K} 2 I<+\infty .
$$

(2) We show that $I$ satisfies the Palais-Smale condition (PS) on $M_{r}$, i.e., for $c \neq 0, \varepsilon>0$ small enough, $u_{n} \in I^{-1}[c-\varepsilon, c+\varepsilon] \cap M_{r}$ and $\left\|I_{M_{r}}^{\prime}\left(u_{n}\right)\right\| \rightarrow 0$, then there is a $u \in M_{r}$ and a subsequence $\left\{u_{n_{j}}\right\}$ such that

$$
\left\|\nabla\left(u_{n_{j}}-u\right)\right\|_{2} \rightarrow 0 \text {. }
$$

Now (2.9) and the boundedness of $\left\{I\left(u_{n}\right)\right\}$ with $\left\{u_{n}\right\} \subseteq M_{r}$ guarantees that $\left\{u_{n}\right\}$ is bounded $W_{0}^{1,2}(\Omega)$, which implies that there exist $u^{*} \in W_{0}^{1,2}(\Omega)$ and subsequence $\left\{u_{n_{j}}\right\}$ of $\left\{u_{n}\right\}$ such that $u_{n_{j}} \rightarrow u^{*}$, as $j \rightarrow+\infty$. Since

$$
\begin{aligned}
I_{M_{r}}^{\prime}(u)(v)= & I^{\prime}(u)(v)-r^{-2} I^{\prime}(u)(u) \int_{\Omega} u v d x \\
= & \left(a+b r^{2}\right) \int_{\Omega} \nabla u \nabla v d x+\int_{\Omega} f(x, u) v d x \\
& -r^{-2}\left(\left(a+b r^{2}\right)\|\nabla u\|_{2}^{2}+\int_{\Omega} f(x, u) u d x\right) \int_{\Omega} u v d x, \quad u, v \in W_{0}^{1,2}(\Omega),
\end{aligned}
$$

we have

$$
\begin{aligned}
& \left(a+b r^{2}\right) \int_{\Omega} \nabla u_{n_{j}} \nabla\left(u_{n_{j}}-u^{*}\right) d x \\
& =I_{M_{r}}^{\prime}\left(u_{n_{j}}\right)\left(u_{n_{j}}-u^{*}\right)-\int_{\Omega} f\left(x, u_{n_{j}}\right)\left(u_{n_{j}}-u^{*}\right) d x \\
& \quad+r^{-2}\left(\left(a+b r^{2}\right)\left\|\nabla u_{n_{j}}\right\|_{2}^{2}+\int_{\Omega} f\left(x, u_{n_{j}}\right) u_{n_{j}} d x\right) \int_{\Omega} u_{n_{j}}\left(u_{n_{j}}-u^{*}\right) d x \\
& \quad \rightarrow 0 .
\end{aligned}
$$


Hence

$$
\left\|\nabla\left(u_{n_{j}}-u^{*}\right)\right\|_{2} \rightarrow 0, \text { as } j \rightarrow+\infty .
$$

(3) We show that $c_{n, r}$ is a critical value of $I(u)$ in $M_{r}$, i.e., there exists a $u_{n, r} \in M_{r}$ such that $c_{n, r}=2 I\left(u_{n, r}\right)$ and $\left.I\right|_{M_{r}} ^{\prime}\left(u_{n, r}\right)=0$.

First, we show that $\forall \varepsilon_{k} \downarrow 0^{+}$, there exists $u_{k} \in 2 I^{-1}\left[c_{n, r}-\varepsilon_{k}, c_{n, r}+\varepsilon_{k}\right]$ such that $I_{M_{r}}^{\prime}\left(u_{k}\right)=0$.

On the contrary, suppose that there is a $\varepsilon_{0}>0$ such that $2 I^{-1}\left[c_{n, r}-\varepsilon_{0}, c_{n, r}+\varepsilon_{0}\right] \cap K=\emptyset, \quad$ where $\quad K=\left\{u \in M_{r}|I|_{M_{r}}^{\prime}(u)=0\right\}$. Let $A_{c}=$ $\{u \mid 2 I(u) \leq c\}$ and $K_{c}=\left\{u|2 I(u)=c, I|_{M_{r}}^{\prime}(u)=\theta\right\}$. From [17], let $N$ be a neighourhood of $K_{c}$, there exists a $\eta(t, u)=\eta_{t}(u) \in C\left([0,1] \times W_{0}^{1,2}(\Omega), W_{0}^{1,2}(\Omega)\right)$ and $\varepsilon_{0}>\varepsilon>0$ such that

(a) $\eta_{0}(u)=u$ for all $u \in W_{0}^{1,2}(\Omega)$;

(b) $\eta_{t}(u)=u$ for all $u \in 2 I^{-1}\left[c_{n, r}-\varepsilon_{0}, c_{n, r}+\varepsilon_{0}\right]$ and for all $t \in[0,1]$;

(c) $\eta_{t}(u)$ is a homeomorphism from $W_{0}^{1,2}(\Omega)$ onto $W_{0}^{1,2}(\Omega)$ for all $t \in[0,1]$;

(d) $I\left(\eta_{t}(u)\right) \leq I(u)$ for all $u \in W_{0}^{1,2}(\Omega)$, for all $t \in[0,1]$;

(e) $\eta_{1}\left(A_{c+\varepsilon}-N\right) \subset A_{c-\varepsilon}$;

(f) If $K_{c}=\emptyset, \eta_{1}\left(A_{c+\varepsilon}\right) \subset A_{c-\varepsilon}$;

(g) If $f$ is even, $\eta_{t}$ is odd in $u$.

Since $c_{n, r}=\inf _{K_{n, r}} \sup _{K} 2 I<+\infty$, for $0<\varepsilon<\varepsilon_{0}$, there is a $A_{n} \subseteq M_{r}$ such that $c_{n, r} \leq \sup _{u \in A_{n}} 2 I(u) \leq c_{n, r}+\varepsilon$. Let $c$ be replaced by $c_{n, r}+\varepsilon$ in the above (a)-(g). It infers from (b) that $\gamma\left(A_{n}\right)=n$ and $\gamma\left(\eta_{1}\left(A_{n}\right)\right)=\gamma\left(A_{n}\right)=n$. Since $2 I^{-1}\left[c_{n, r}-\varepsilon_{0}, c_{n, r}+\varepsilon_{0}\right] \cap K=$ $\emptyset$ and $\varepsilon<\varepsilon_{0}$, from (f), we have $\eta_{1}\left(A_{c_{n, r}+\varepsilon}\right) \subset A_{c_{n, r}-\varepsilon}$, which together with $A_{n} \subset 2 I^{-1}\left[c_{n, r}-\right.$ $\left.\varepsilon, c_{n, r}+\varepsilon\right] \subseteq A_{c_{n, r}+\varepsilon}$ guarantees that $\eta_{1}\left(A_{n}\right) \subset A_{c_{n, r}-\varepsilon}$ also. Hence,

$$
c_{n, r}=\inf _{K_{n, r}} \sup _{K} 2 I \leq \sup _{u \in \eta_{1}\left(A_{n}\right)} 2 I(u) \leq c_{n, r}-\varepsilon .
$$

This is contradiction.

Second, obviously, $\left\{I\left(u_{k}\right)\right\}$ is bounded and $\left\{I_{M_{r}}^{\prime}\left(u_{k}\right)=0\right\}$. The Palais-Smale condition implies that $\left\{u_{k}\right\}$ has a convergent subsequence. Without loss of generality, we assume that

$$
u_{k} \rightarrow u_{n, r}, \quad k \rightarrow+\infty .
$$

It is easy to see that $u_{n, r} \in M_{r}$ such that

$$
c_{n, r}=2 I\left(u_{n, r}\right)
$$

and

$$
I^{\prime}\left(u_{n, r}\right)(v)=r^{-2} I^{\prime}\left(u_{n, r}\right)\left(u_{n, r}\right) \cdot u_{n, r}(v), \forall v \in W_{0}^{1,2}(\Omega) .
$$

Let $\mu_{n, r}=r^{-2} I^{\prime}\left(u_{n, r}\right)\left(u_{n, r}\right)$. Note one has

$$
\left(a+b r^{2}\right) \int_{\Omega} \nabla u_{n, r} \nabla v d x+\int_{\Omega} f\left(x, u_{n, r}\right) v(x) d x=\mu_{n, r} \int_{\Omega} u_{n, r} v d x, \forall v \in W_{0}^{1,2}
$$

By $u_{n, r} \in M_{r}$, (2.6) becomes 


$$
\left(a+b \int_{\Omega} u_{n, r}^{2} d x\right) \int_{\Omega} \nabla u_{n, r} \nabla v d x+\int_{\Omega} f\left(x, u_{n, r}\right) v(x) d x=\mu_{n, r} \int_{\Omega} u_{n, r} v d x, \forall v \in W_{0}^{1,2}
$$

i.e. problem (1.1) has a sequence eigenvalues $\left\{\mu_{n, r}\right\}$ with corresponding eigenfunctions $\left\{u_{n, r}\right\}$. Let $v=u_{n, r}$. Then (2.10) becomes

$$
r^{2} \mu_{n, r}=\left(a+b r^{2}\right)\left\|\nabla u_{n, r}\right\|_{2}^{2}+\int_{\Omega} f\left(x, u_{n, r}\right) u_{n, r} d x .
$$

The proof is completed.

Corollary 2.1 Let $f \equiv 0$ and equation (1.1) becomes

$$
\begin{array}{cc}
-\left(a+b\|u\|_{2}^{2}\right) \Delta u=\lambda u, & x \text { in } \Omega, \\
u(x)=0, \quad x \text { on } \partial \Omega . & (2.11)_{\lambda}
\end{array}
$$

Then, $(2.11)_{\lambda}$ has branches

$$
\left.C_{n}=\left\{\left(a+b r^{2}\right) \lambda_{n}, \pm r v_{n}\right) \mid r>0\right\}, \quad n=1,2, \ldots
$$

Proof From the L-S procedure in Theorem 2.1, $(2.11)_{\lambda}$ has exactly the eigenvalues $\mu_{n, r}^{0}$ with the corresponding eigenfunction $u_{n, r}^{0}\left(\left\|u_{n, r}^{0}\right\|_{2}=r\right)$ which satisfies

$$
\left\{\begin{array}{r}
-\Delta u_{n, r}^{0}=\mu_{n, r}^{0} \frac{1}{a+b\left\|u_{n, r}^{0}\right\|_{2}^{2}} u_{n, r}^{0}=\mu_{n, r}^{0} \frac{1}{a+b r^{2}} u_{n, r}^{0}, \quad x \text { in } \Omega, \\
u_{n, r}^{0}(x)=0, \quad x \text { on } \partial \Omega .
\end{array}\right.
$$

Comparing $(2.11)_{\lambda}$ with $(2.1)_{\lambda}$, we get

$$
\mu_{n, r}^{0} \frac{1}{a+b r^{2}}=\lambda_{n}
$$

and $u_{n, r}^{0}=k_{n} u_{n}$, where $u_{n}$ is the corresponding eigenvalue function to $\lambda_{n}$ of $(2.1)_{\lambda}$ with $\left\|u_{n}\right\|_{2}=r$. Moreover,

$$
c_{n, r}^{0}=2 \Phi\left(u_{n, r}^{0}\right)=\left(a+b r^{2}\right)\left\|\nabla u_{n, r}^{0}\right\|_{2}^{2}, \quad \mu_{n, r}^{0}=\left(a+b r^{2}\right) \lambda_{n} .
$$

Since $u_{n, r}^{0}=k_{n} u_{n}$, one has

$$
r=\left\|u_{n, r}^{0}\right\|_{2}=\left\|k_{n} u_{n}\right\|_{2}=\left|k_{n}\right| r,
$$

which implies $k_{n}= \pm 1$ and $u_{n, r}^{0}= \pm u_{n}$. Hence, (2.12) becomes

$$
c_{n, r}^{0}=2 \Phi\left(u_{n, r}^{0}\right)=\left(a+b r^{2}\right)\left\|\nabla u_{n}\right\|_{2}^{2}, \quad \mu_{n, r}^{0}=\left(a+b r^{2}\right) \lambda_{n} .
$$

From (2.2), we have

$$
r^{2} \lambda_{n}=\int_{\Omega}\left|\nabla u_{n}\right|^{2} d x=\left\|\nabla u_{n}\right\|_{2}^{2}=\left\|\nabla u_{n, r}^{0}\right\|_{2}^{2},
$$


and so

$$
c_{n, r}^{0}=2 \Phi\left(u_{n, r}^{0}\right)=\left(a+b r^{2}\right) r^{2} \lambda_{n}, \quad \mu_{n, r}^{0}=\left(a+b r^{2}\right) \lambda_{n},
$$

which together with (2.3) implies that $(2.11)_{\lambda}$ has branches

$$
\left.C_{n}=\left\{\left(a+b r^{2}\right) \lambda_{n}, \pm r v_{n}\right) \mid r>0\right\}, \quad n=1,2, \ldots
$$

The proof is completed.

\section{Bifurcation results concerning the eigenvalues of some related linear problem to (1.1)}

In Sect. 2, we obtained the branches of solutions of (1.1) when $f \equiv 0$. Now we consider the case $f \not \equiv 0$.

Theorem 3.1 Let the assumptions of Theorem 2.1 be satisfied with $p>1$ and $d=0$ in the growth assumption $\left(A_{1}\right)$. Then each a $\lambda_{n}$ is a bifurcation point (in $W_{0}^{1,2}(\Omega)$ ) for (1.1); more precisely, for each $n=1,2, \cdots$, the eigenvalue-eigenfunction pairs $\left(\mu_{n, r}, u_{n, r}\right)$ given by Theorem 2.1 satisfy $\mu_{n, r}=a \lambda_{n}+b \lambda_{n} r^{2}+O\left(r^{\min \{2, p-1\}}\right)$ as $r \rightarrow 0$.

Proof Let $\gamma=p-1$. Then (see Lemma 2.1) we have

$$
\|u\|_{p+1}^{p+1} \leq c\|\nabla u\|_{2}^{2}\|u\|_{2}^{p-1}, u \in W_{0}^{1,2}(\Omega) .
$$

Note $\left(d=0\right.$ in $\left.\left(A_{1}\right)\right)$

$$
|I(u)-\Phi(u)|=\left|\int_{\Omega} F(x, u) d x\right| \leq c \int_{\Omega}|u|^{p+1} d x .
$$

Since

$$
\Phi(u)=\left(a+b r^{2}\right)\|u\|^{2}
$$

from (3.1), we have

$$
\int_{\Omega}|u|^{p+1} d x \leq c \frac{1}{a+b r^{2}} \Phi(u)\|u\|_{2}^{p-1} \leq \frac{c}{a} \Phi(u)\|u\|_{2}^{p-1} .
$$

Hence,

$$
\int_{\Omega}|u|^{p+1} d x \leq \frac{c}{a} \Phi(u) r^{p-1}, \quad \forall u \in M_{r}
$$

It infers from (3.2) that

$$
\left(1-\frac{c}{a} r^{p-1}\right) \Phi(u) \leq I(u) \leq\left(1+\frac{c}{a} r^{p-1}\right) \Phi(u),
$$

and so

$$
\left(1-\frac{c}{a} r^{p-1}\right) \inf _{K_{n, r}} \sup _{K} 2 \Phi(u) \leq \inf _{K_{n, r}} \sup _{K} 2 I(u) \leq\left(1+\frac{c}{a} r^{p-1}\right) \inf _{K_{n, r}} \sup _{K} 2 \Phi(u),
$$


i.e.

$$
\left(1-\frac{c}{a} r^{p-1}\right) c_{n, r}^{0} \leq c_{n, r} \leq\left(1+\frac{c}{a} r^{p-1}\right) c_{n, r}^{0}
$$

which implies that

$$
\left|c_{n, r}-c_{n, r}^{0}\right| \leq \frac{c}{a} c_{n, r}^{0} r^{p-1} .
$$

Now (2.9) guarantees that

$$
\left|c_{n, r}^{0}\right| \leq c r^{2}
$$

and so

$$
\left|c_{n, r}-c_{n, r}^{0}\right| \leq c r^{p+1} .
$$

It deduces from Theorem 2.1 and (3.2) that

$$
\begin{aligned}
c_{n, r} & =\left(a+b r^{2}\right)\left\|\nabla u_{n, r}\right\|_{2}^{2}+2 \int_{\Omega} F\left(x, u_{n, r}\right) d x \\
& \geq\left(a+b r^{2}\right)\left\|\nabla u_{n, r}\right\|_{2}^{2}-2 c r^{p-1}\left\|\nabla u_{n, r}\right\|_{2}^{2} \\
& =\left(a+b r^{2}-2 c r^{p-1}\right)\left\|\nabla u_{n, r}\right\|_{2}^{2},
\end{aligned}
$$

which together with (3.3) implies that

$$
\begin{aligned}
\left\|\nabla u_{n, r}\right\|_{2}^{2} & \leq \frac{c_{n, r}}{a+b r^{2}-2 c r^{p-1}} \\
& \leq \frac{c_{n, r}^{0}+c r^{p+1}}{a+b r^{2}-2 c r^{p-1}} \\
& \leq c r^{2} .
\end{aligned}
$$

From Theorem 2.1 and (3.1), (3.4), one has

$$
\begin{aligned}
\left|c_{n, r}-r^{2} \mu_{n, r}\right| & =\left|2 \int_{\Omega} F\left(x, u_{n, r}\right) d x-\int_{\Omega} f\left(x, u_{n, r}\right) u_{n, r} d x\right| \\
& \leq c\left\|\nabla u_{n, r}\right\|_{2}^{2 \alpha} r^{\beta}+d r \\
& \leq c\left(c_{n, r}\right)^{\alpha}+d r \\
& \leq c\left(c_{n, r}^{0}+c r^{\beta}\left(c_{n, r}^{0}\right)^{\alpha}+d r\right)^{\alpha}+d r,
\end{aligned}
$$

Then

$$
\begin{aligned}
\left|r^{2} \mu_{n, r}-r^{2} \mu_{n, r}^{0}\right| & =\left|r^{2} \mu_{n, r}-c_{n, r}+c_{n, r}-c_{n, r}^{0}+c_{n, r}^{0}-r^{2} \mu_{n, r}^{0}\right| \\
& \leq\left|r^{2} \mu_{n, r}-c_{n, r}\right|+\left|c_{n, r}-c_{n, r}^{0}\right|+\left|c_{n, r}^{0}-r^{2} \mu_{n, r}^{0}\right| \\
& \leq c_{1} r^{p+1}+c_{2} r^{p+1}+c_{3} r^{4} \\
& \leq c r^{\min \{4, p+1\}},
\end{aligned}
$$

which implies that

$$
\left|\mu_{n, r}-\mu_{n, r}^{0}\right| \leq c r^{\min \{2, p-1\}} .
$$


Consequently,

$$
\mu_{n, r}=a \lambda_{n}+b \lambda_{n} r^{2}+O\left(r^{\min \{2, p-1\}}\right)
$$

The proof is completed.

\section{The asymptotic distribution of the eigenvalue $\mu_{n, r}$ of (1.1)}

In this section, we consider the asymptotic laws of the eigenvalue $\mu_{n, r}$ of (1.1).

Lemma 4.1 Assume $\left(A_{1}\right)$ holds. For $r>0$ and $n=1,2, \ldots$, let $\mu_{n, r}, c_{n, r}$ be as in Theorem 2.1 , and let $\lambda_{n}$ be the eigenvalues of the linear problem $(2.1)_{\lambda}$. Then

$$
\left|c_{n, r}-c_{n, r}^{0}\right| \leq c r^{\beta}\left(c_{n, r}^{0}\right)^{\alpha}+d r
$$

and

$$
\left|c_{n, r}-r^{2} \mu_{n, r}\right| \leq c\left(c_{n, r}^{0}+c r^{\beta}\left(c_{n, r}^{0}\right)^{\alpha}+d r\right)^{\alpha}+d r,
$$

where $\alpha=(p-1) N / 4$ and $\beta=(p+1)-(p-1) N / 2$; here and henceforth $\mathrm{c}, \mathrm{d}$ denote some, but not always the same, positive constants.

Proof First notice that the growth assumption $\left(A_{1}\right)$ implies

$$
\left|\int_{\Omega} F(x, u) d x\right| \leq c \int_{\Omega}|u|^{p+1} d x+d \int_{\Omega}|u| d x
$$

and similarly

$$
\left|\int_{\Omega} f(x, u) u d x\right| \leq c \int_{\Omega}|u|^{p+1} d x+d \int_{\Omega}|u| d x .
$$

Next, as $1 \leq p<\bar{p}$, from Lemma 2.1, if $\int_{\Omega} u^{2} d x=r^{2}$, we have

$$
\begin{aligned}
\left|\int_{\Omega} F(x, u) d x\right| & \leq c\|\nabla u\|_{2}^{2 \alpha} r^{\beta}+d r \\
e & =c\left(\frac{1}{a+b r^{2}}\left(a+b r^{2}\right)\|\nabla u\|_{2}^{2}\right)^{\alpha} r^{\beta}+d r \\
& \leq c\left(\frac{1}{a}\right)^{\alpha}(\Phi(u))^{\alpha} r^{\beta}+d r
\end{aligned}
$$

and similarly

$$
\left|\int_{\Omega} f(x, u) u d x\right| \leq c\left(\frac{1}{a}\right)^{\alpha}(\Phi(u))^{\alpha} r^{\beta}+d r,
$$

with $\alpha$ and $\beta$ as in the statement of Lemma 4.1.

To prove (4.1), observe that (4.3) implies 


$$
\begin{aligned}
I(u) & =\Phi(u)+\int_{\Omega} F(x, u) d x \\
& \leq \Phi(u)+c r^{\beta}(\Phi(u))^{\alpha}+d r
\end{aligned}
$$

holds ( $c$ instead of $c\left(\frac{1}{a}\right)^{\alpha}$ ). In other words, we have

$$
I(u) \leq g(\Phi(u))
$$

where $g: R^{+} \rightarrow R^{+}$is defined by

$$
g(t)=t+c r^{\beta} t^{\alpha}+d r .
$$

As $g$ is continuous and nondecreasing, we get

$$
\inf _{K_{n, r}} \sup _{K \in K_{n, r}} I(u) \leq \inf _{K_{n, r}} \sup _{K \in K_{n, r}} g(\Phi(u))=g\left(\inf _{K_{n, r}} \sup _{K \in K_{n, r}} \Phi(u)\right) .
$$

Now Theorem 2.1 implies that

$$
c_{n, r} \leq 2 g\left(c_{n, r}^{0}\right)=c_{n, r}^{0}+c r^{\beta}\left(c_{n, r}^{0}\right)^{\alpha}+d r
$$

for some new constants $c$ and $d>0$. Therefore,

$$
\left|c_{n, r}-c_{n, r}^{0}\right| \leq c r^{\beta}\left(c_{n, r}^{0}\right)^{\alpha}+d r,
$$

which shows (4.1) is true.

Since

$$
c_{n, r}=\left(a+b r^{2}\right)\left\|u_{n, r}\right\|_{2}^{2}+2 \int_{\Omega} F(x, u) d x
$$

we have

$$
\left(a+b r^{2}\right)\left\|\nabla u_{n, r}\right\|_{2}^{2}=c_{n, r}-2 \int_{\Omega} F\left(x, u_{n, r}\right) d x .
$$

It deduces from Theorem 2.1 and (4.3)-(4.4) that

$$
\begin{aligned}
\left|c_{n, r}-r^{2} \mu_{n, r}\right| & =\left|2 \int_{\Omega} F\left(x, u_{n, r}\right) d x-\int_{\Omega} f\left(x, u_{n, r}\right) u_{n, r} d x\right| \\
& \leq c\left\|\nabla u_{n, r}\right\|_{2}^{2 \alpha} r^{\beta}+d r \\
& \leq c\left(c_{n, r}\right)^{\alpha}+d r \\
& \leq c\left(c_{n, r}^{0}+c r^{\beta}\left(c_{n, r}^{0}\right)^{\alpha}+d r\right)^{\alpha}+d r,
\end{aligned}
$$

which completes the proof of the lemma.

Lemma 4.2 (Theorem 2, [5]) The eigenvalues $\lambda_{n}$ of $(2.1)_{\lambda}$ satisfy, as $n \rightarrow+\infty$

$$
\lambda_{n}=k n^{2 / N}+O\left(n^{1 / N} \log n\right), \quad n=1,2, \cdots,
$$

where

$$
k=(2 \pi)^{2}(V)^{-2 / N}
$$

and $V$ is the value of $B(\theta, 1)$. 
Theorem 4.1 Assume that $\left(A_{1}\right)$ holds. Then given any $r>0,(1.1)$ has infinitely many eigenfunctions $u_{n, r}(n=1,2, \ldots)$ with $\int_{\Omega} u_{n, r}^{2} d x=r^{2}$, whose corresponding eigenvalues $\mu_{n, r}$ satisfy, as $n \rightarrow+\infty$ and with $k$ as in (4.7),

$$
\mu_{n, r}=\left(a+b r^{2}\right) k n^{2 / N}+O\left(n^{1 / N} \log n\right),
$$

where $\bar{p}$ is defined in $\left(A_{1}\right)$.

Proof Since condition $\left(A_{1}\right)$ is true, Theorem 2.1 guarantees that for given any $r>0,(1.1)$ has infinitely many eigenfunctions $u_{n, r}(n=1,2, \ldots)$ with $\int_{\Omega} u_{n, r}^{2} d x=r^{2}$.

Now $p<\bar{p}=\min \left\{2^{*}-1,1+4 / N\right\}$ guarantees that $\alpha=(p-1)(N / 4)<1$. Thus, (4.3) guarantees that

$$
\begin{aligned}
c_{n, r} & =c_{n, r}^{0}+O\left(\left(c_{n, r}^{0}\right)^{\alpha}\right) \\
& =\left(a+b r^{2}\right) r^{2} \lambda_{n}+O\left(\left(c_{n, r}^{0}\right)^{\alpha}\right) \\
& =\left(a+b r^{2}\right) r^{2} \lambda_{n}+O\left(\lambda_{n}^{\alpha}\right)
\end{aligned}
$$

and

$$
\begin{aligned}
\left(c_{n, r}\right)^{\alpha} & =\left(\left(a+b r^{2}\right) r^{2} \lambda_{n}+O\left(\lambda_{n}^{\alpha}\right)\right)^{\alpha} \\
& =O\left(\lambda_{n}^{\alpha}\right), \\
\left(c_{n, r}\right)^{\frac{1}{2}} & =O\left(\lambda_{n}^{\frac{1}{2}}\right),
\end{aligned}
$$

which together (4.1) and (4.2) implies that

$$
\begin{aligned}
\left|r^{2} \mu_{n, r}-c_{n, r}^{0}\right| & =\left|r^{2} \mu_{n, r}-c_{n, r}+c_{n, r}-c_{n, r}^{0}\right| \\
& \leq\left|r^{2} \mu_{n, r}-c_{n, r}\right|+\left|c_{n, r}-c_{n, r}^{0}\right| \\
& \leq c\left(c_{n, r}^{0}\right)^{\alpha}+c r^{\beta}\left(c_{n, r}^{0}\right)^{\alpha} \\
& =O\left(\lambda_{n}^{\alpha}\right),
\end{aligned}
$$

and so

$$
r^{2} \mu_{n, r}=c_{n, r}^{0}+O\left(\lambda_{n}^{\alpha}\right)=r^{2}\left(a+b r^{2}\right) \lambda_{n}+O\left(\lambda_{n}^{\alpha}\right)
$$

Consequently

$$
\mu_{n, r}=\left(a+b r^{2}\right) \lambda_{n}+O\left(\lambda_{n}^{\alpha}\right)
$$

Since

$$
\lambda_{n}=k n^{2 / N}+O\left(n^{1 / N} \log n\right)
$$

we have

$$
\begin{aligned}
\mu_{n, r} & =\left(a+b r^{2}\right) k n^{2 / N}+O\left(n^{1 / N} \log n\right)+O\left(\left(k n^{2 / N}+O\left(n^{1 / N} \log n\right)\right)^{\alpha}\right) \\
& =\left(a+b r^{2}\right) k n^{2 / N}+O\left(n^{1 / N} \log n\right) .
\end{aligned}
$$

The proof is completed. 
Open Access This article is licensed under a Creative Commons Attribution 4.0 International License, which permits use, sharing, adaptation, distribution and reproduction in any medium or format, as long as you give appropriate credit to the original author(s) and the source, provide a link to the Creative Commons licence, and indicate if changes were made. The images or other third party material in this article are included in the article's Creative Commons licence, unless indicated otherwise in a credit line to the material. If material is not included in the article's Creative Commons licence and your intended use is not permitted by statutory regulation or exceeds the permitted use, you will need to obtain permission directly from the copyright holder. To view a copy of this licence, visit http://creativecommons.org/licenses/by/4.0/.

\section{References}

1. Alves, C.O., Corrêa, F.J.S.A.: On existence of solutions for a class of problems involving a nonlinear operator. Comm. Appl. Nonlinear Anal. 8(2), 43-56 (2001)

2. Alves, C.O., Corrêa, F.J.S.A., Figueiredo, G.M.: On a class of nonlocal elliptic problems with critical growth. Differ. Equ. Appl. 2, 409-417 (2010)

3. Alves, C.O., Covei, D.P.: Existence of solution for a class of nonlocal elliptic problem via subsupersolution method. Nonlinear Anal. Real World Appl. 23, 1-8 (2015)

4. Amann, H.: Lusternik-Schnirelman theory and non-linear Eigenvalue Problems. Math. Ann. 199, 55-72 (1972)

5. Bruning, J.: Zur abschatzung der spektralfunction elliptischer operatoren. Math. Z. 137, 75-85 (1974)

6. Carrier, G.F.: On the nonlinear vibration problem of the elastic string. Q. J. Appl. Math. 3(2), 157-165 (1945)

7. Chiappinelli, R.: Remarks on bifurcation for elliptic operator with odd nonlinearity. Israel J. Math. 65(3), 285-293 (1989)

8. Chiappinelli, R.: On spectral asymptotics and bifurcation for elliptic operators with odd superlinearity term. Nonlinear Anal. Theory Methods Appl. 13(7), 871-878 (1989)

9. Chipot, M., Lovat, B.: Some remarks on nonlocal elliptic and parabolic problems. Nonlinear Anal. 30, 4619-4627 (1997)

10. Chipot, M., Corrêa, F.J.S.A.: Boundary layer solutions to functional elliptic equations. Bull. Braz. Math. Soc. New Ser. 40(3), 381-393 (2009)

11. Corrêa, F.J.S.A.: On positive solutions of nonlocal and nonvariational elliptic problems. Nonlinear Anal. 59, 1147-1155 (2004)

12. Corrêa, F.J.S.A., Menezes, S.D.B., Ferreira, J.: On a class of problems involving a nonlocal operator. Appl. Math. Comput. 147, 475-489 (2004)

13. do Ó, J. M., Lorca, S., Sánchez, J., Ubilla, P.: Positive solutions for some nonlocal and nonvariational elliptic systems. Complex Var. Elliptic Equ. 61(3), 1-18 (2016)

14. Figueiredo-Sousa, T.S., Morales-Rodrigo, C., Suárez, A.: Some superlinear problems with nonlocal diffusion coefficient. J. Math. Anal. Appl. 482(1) , 123519, 25pp (2020)

15. Li, X., Wu, J.: Stability of nonlinear differential systems with state-dependent delayed impulses. Automatica 64, 63-69 (2016)

16. Li, X., Song, S.: Stabilization of Delay Systems: Delay-dependent Impulsive Control. IEEE Trans Autom. Control 62(1), 406-411 (2017)

17. Prodi, G.: Eigenvalues of non-linear problems. Cremonese, Roma (1974)

18. Shibata, T.: Asymptotic properties of variational eigenvalues for semilinear elliptic operators. Boll. Un. Mat. Ital. 7(2B), 411-426 (1988)

19. Shibata, T.: Precise asymptotic formulas for semilinear eigenvalue problems. Ann. Henri Poincaré 2 , 713-732 (2001)

20. Shibata, T.: Precise spectral asymptotics for nonlinear Sturm-Liouville problems. J. Differ. Equ. 180, 374-394 (2002)

21. Shibata, T.: Global behavior of the branch of positive solutions to a logistic equation of population dynamics. Proc. Am. Math. Soc. 136(7), 2547-2554 (2008)

22. Shuai, W.: Sign-changing solutions for a class of Kirchhoff-type problem in bounded domains. J. Differ. Equ. 259, 1256-1274 (2015)

23. Stuart, C.A.: Bifurcation from the continuous spectrum in $L^{2}$-theory of elliptic equations on $R^{n}$. In Recenl Methods in Nonlinear Analysis and Applications, Liguori, Napoli (1981)

24. Stuart, C.A.: Bifurcation in $L^{p}\left(R^{N}\right)$ for a semilinear elliptic equation. Proc. Lond. Math. Sot. 57(3), 511-541 (1988) 
25. Stuart, C.A.: Bifurcation from the essential spectrum for some non-compact nonlinearities. Math. Appl. Sci. 11, 525-542 (1989)

26. Yan, B., Wang, D.: The multiplicity of positive solutions for a class of nonlocal elliptic problem. J. Math. Anal. Appl. 442(1), 72-102 (2016)

27. Yan, B., Ma, T.: The existence and multiplicity of positive solutions for a class of nonlocal elliptic problems. Bound. Value Probl. 165, 1-35 (2016)

28. Yan, B., Ren, Q.: Existence, uniqueness and multiplicity of positive solutions for some nonlocal singular elliptic problems. Electron. J. Differ. Equ. 2017(138), 1-21 (2017) 